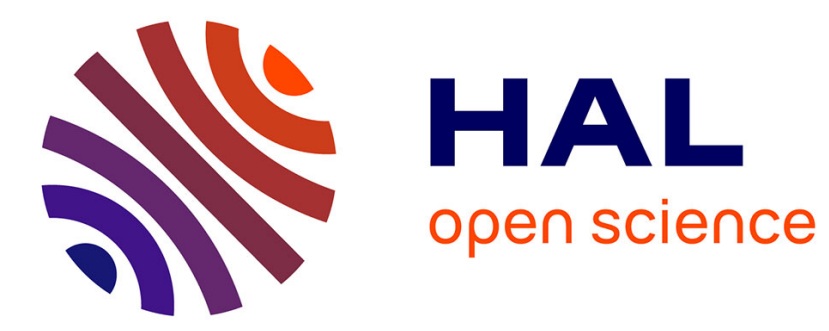

\title{
Local error of a splitting scheme for a nonlinear Schrödinger-type equation with random dispersion \\ Renaud Marty
}

\section{To cite this version:}

Renaud Marty. Local error of a splitting scheme for a nonlinear Schrödinger-type equation with random dispersion. 2019. hal-02314915

\section{HAL Id: hal-02314915 \\ https://hal.univ-lorraine.fr/hal-02314915}

Preprint submitted on 14 Oct 2019

HAL is a multi-disciplinary open access archive for the deposit and dissemination of scientific research documents, whether they are published or not. The documents may come from teaching and research institutions in France or abroad, or from public or private research centers.
L'archive ouverte pluridisciplinaire HAL, est destinée au dépôt et à la diffusion de documents scientifiques de niveau recherche, publiés ou non, émanant des établissements d'enseignement et de recherche français ou étrangers, des laboratoires publics ou privés. 


\title{
Local error of a splitting scheme for a nonlinear Schrödinger-type equation with random dispersion
}

\author{
Renaud Marty*
}

\begin{abstract}
We study a Lie splitting scheme for a nonlinear Schrödinger-type equation with random dispersion. The main result is an approximation of the local error. Then we can deduce sharp order estimates, for instance in the case of a white noise dispersion.
\end{abstract}

Key-words: Splitting schemes, nonlinear Schrödinger-type equations, stochastic partial differential equations.

\section{Introduction}

We consider the nonlinear Schrödinger equation with white noise dispersion which has been recently studied. It can be written as

$$
u(t, x)=u_{0}(x)+i \int_{0}^{t} \frac{\partial^{2} u}{\partial x^{2}}(\theta, x) \circ d W(\theta)+\int_{0}^{t} g(u(\theta, x)) d \theta, \quad(t, x) \in[0,1] \times \mathbb{R} .(1)
$$

where $u_{0} \in L^{2}(\mathbb{R}, \mathbb{C}), W$ is a Brownian motion and $g$ is a complex nonlinear function. The existence and the uniqueness of the solution are established for instance for a Lipschitz nonlinearity in [9], for a cubic nonlinearity in [6], and for a quintic nonlinearity in [7]. Regarding its numerical analysis, several schemes have been studied (for example in $[3,5,9])$. In particular, a Lie splitting scheme is analyzed in [9]. Among other results, it is proven that the order of the scheme is at least $1 / 2$ and numerical experiments show that this order is 1 (also shown in [5] and [3]). In addition, order estimates have been established when the Brownian motion $W$ in (1) is replaced by another process. For instance, if $W$ is an $\alpha$-Hölder function $(\alpha \in(0,1))$ then the order is $\alpha([9])$, or if $W$ is a fractional Brownian motion with Hurst index $H \in(0,1)$ then the order is $H([8])$. As in many other works dealing with splitting schemes for other deterministic or random equations (see for instance $[10,4,1,2,8,9]$ ), the study of the order crucially involves estimates of the local error.

In this paper we consider a Lie splitting scheme for a nonlinear Schrödinger-type equation with random dispersion. This equation has the same form as (1) but $W$ is a

\footnotetext{
*Institut Élie Cartan de Lorraine, Université de Lorraine, B.P. 70239, F-54506 Vandoeuvre-lès-Nancy Cedex, France. Email: renaud.marty@univ-lorraine.fr
} 
continuous random process and $i \partial^{2} / \partial x^{2}$ is replaced by a more general operator. Under assumptions on the moments of the random dispersion term, we establish an expansion of the local error, which gives us more information than the usual estimates. As a corollary, if the dispersion term is defined from a process with independent increments and mean zero, we deduce a sharp order estimate. In the particular case of a white noise dispersion, we show that the order of the scheme is 1 . This has been theoretically or numerically observed for splitting or other schemes (for instance [3, 5, 9]) for Equation (1).

The paper is organized as follows. We present the framework in Section 2. The main result about the approximation of the local error is given in Section 3. Section 4 is devoted to the proofs. In Section 5 we discuss an application to order estimates. In Section 6 we study another splitting scheme.

Throughout the paper we denote by $L^{2}$ the space $L^{2}(\mathbb{R}, \mathbb{C})$, by $\|\cdot\|_{L^{2}}$ its norm and by $\langle\cdot, \cdot\rangle_{L^{2}}$ its inner product. For every $\sigma \in \mathbb{N}$, we denote by $H^{\sigma}$ the Sobolev space of all function $f \in L^{2}$ such that $\xi \mapsto\left(1+|\xi|^{2}\right)^{\sigma / 2} \mathcal{F}(f)(\xi) \in L^{2}$ where $\mathcal{F}$ is the Fourier transform. The norm is denoted by $\|\cdot\|_{H^{\sigma}}$ and the inner product by $\langle\cdot, \cdot\rangle_{H^{\sigma}}$.

In this paper we consider a probability space $(\Omega, \mathcal{T}, \mathbb{P})$. The expectation is $\mathbb{E}$ and if $q \geq 1$ and $M$ is random variable taking its values in a normed space $F=\left(F,\|\cdot\|_{F}\right)$, we denote $\sqrt[q]{\mathbb{E}\left[\|M\|_{F}^{q}\right]}$ by $\|M\|_{L_{\Omega}^{q}(F)}$ when it is well defined.

\section{Framework}

\subsection{Nonlinear Schrödinger-type equation with random dispersion}

Let $u_{0}$ be function in $L^{2}, g$ be a function from $\mathbb{C}$ to $\mathbb{C}, a$ be a function from $\mathbb{R}$ to $\mathbb{R}$ and $W$ be a continuous stochastic process. We assume that there exist $C_{a}>0$ and $\sigma \in \mathbb{N}$ such that for all $\xi \in \mathbb{R}$,

$$
|a(\xi)| \leq C_{a}\left(1+|\xi|^{2}\right)^{\sigma / 2} .
$$

We consider the following nonlinear Schrödinger-type equation

$$
u(t, x)=u_{0}(x)+\int_{0}^{t} A u(\theta, x) \circ d W(\theta)+\int_{0}^{t} g(u(\theta, x)) d \theta, \quad(t, x) \in[0,1] \times \mathbb{R}
$$

where $\circ d W$ stands for a Stratonovich-type differentiation we explain below and $A$ is the operator on $H^{\sigma}$ such that for all $f \in H^{\sigma}$,

$$
A f=\mathcal{F}^{-1}(\xi \rightarrow i a(\xi) \mathcal{F}(f)(\xi))
$$

where $\mathcal{F}$ is the Fourier transform with respect to $x$. We define

$$
\Delta=\left\{(s, t) \in[0,1]^{2} \mid s \leq t\right\} .
$$

Equation (3) is rewritten as

$$
u(t, x)=X(0, t) u_{0}(x)+\int_{0}^{t} X(\theta, t) g(u(\theta, x)) d \theta, \quad(t, x) \in[0,1] \times \mathbb{R}
$$


where the family of operators $\left\{X\left(t_{0}, t\right)\right\}_{\left(t_{0}, t\right) \in \Delta}$ is defined such that for every $v \in L^{2}$ and every $(t, x) \in[0,1] \times \mathbb{R}$,

$$
X\left(t_{0}, t\right) v(x)=\mathcal{F}^{-1}\left(\xi \rightarrow \exp \left(i a(\xi)\left(W(t)-W\left(t_{0}\right)\right)\right) \mathcal{F}(v)(\xi)\right)(x) .
$$

Notice that for all $p \in \mathbb{N},\left\|X\left(t_{0}, t\right) v\right\|_{H^{p}}=\|v\|_{H^{p}}$ because $a$ and $W$ are real.

Throughout the paper, when $g:(\Re z, \Im z) \mapsto g(z)$ is considered as a function of two real variables, we assume that for all $k \in \mathbb{N}^{*}$ its partial derivatives of order $k$ exist and are bounded. This implies that $g$ and all its derivatives are Lipschitz functions. Therefore, for every $k \in \mathbb{N}^{*}$ its differential of order $k$ denoted by $\nabla^{k} g$ is bounded. Moreover, we assume that $g(0)=0$ and $(\nabla g)(0)=(0,0)$.

Under these assumptions, we can prove (as in [9] for instance) that there exists a unique solution to (5) denoted by $u$ and almost surely in $\mathcal{C}\left([0,1], L^{2}\right)$. We denote by $S=\left\{S\left(t_{0}, t\right)\right\}_{\left(t_{0}, t\right) \in \Delta}$ the family of operators on $L^{2}$ satisfying $S\left(t_{0}, t_{2}\right)=S\left(t_{1}, t_{2}\right) S\left(t_{0}, t_{1}\right)$ if $t_{0} \leq t_{1} \leq t_{2}$ and such that the solution $u$ is given by $u(t, x)=S(0, t) u_{0}(x)$. Moreover, we denote by $Y=\{Y(t)\}_{t \in[0,1]}$ the family of operators such that for every $u_{0}$ the function $w:(t, x) \mapsto w(t, x)=Y(t) u_{0}$ is solution of

$$
w(t, x)=u_{0}(x)+\int_{0}^{t} g(w(\theta, x)) d \theta, \quad(t, x) \in[0,1] \times \mathbb{R} .
$$

\subsection{Lie splitting scheme}

We define

$$
\delta:=\{(n, h) \in \mathbb{N} \times(0,1] \mid n h \leq 1\} .
$$

For all $t_{0} \in[0,1]$ we denote by $u_{t_{0}}$ the (random) function $u\left(t_{0}, \cdot\right): x \mapsto u\left(t_{0}, x\right)$. In order to approximate $u_{n h}$ for all $(n, h) \in \delta$, we define the (Lie) splitting scheme as

$$
u_{n}^{h}:=Z_{n}^{h} \cdots Z_{1}^{h} u_{0}
$$

where

$$
Z_{k}^{h}:=Z((k-1) h, k h) \text { for all } k \in\{1, \cdots, n\}
$$

and

$$
Z\left(t_{0}, t\right):=Y\left(t-t_{0}\right) X\left(t_{0}, t\right) \text { for all }\left(t_{0}, t\right) \in \Delta .
$$

We also define

$$
S_{k}^{h}:=S((k-1) h, k h) \text { for all } k \in\{1, \cdots, n\}
$$

such that

$$
u_{n h}:=S_{n}^{h} \cdots S_{1}^{h} u_{0} .
$$

We fix a real number $\beta \geq 1$. We assume that $W$ is a stochastic process with continuous sample paths and finite moments of order $\beta$, and such that there exist $\alpha \in(0,1]$ and $C>0$ such that for all $(s, t)$,

$$
\mathbb{E}\left[|W(t)-W(s)|^{\beta}\right] \leq C|t-s|^{\beta \alpha} .
$$

The following theorem generalizes error estimates proven in [9]. 
Theorem 2.1. Let $m \in \mathbb{N}$. If $u_{0} \in H^{m+\sigma}$ then there exists a constant $C>0$ such that for all $(n, h) \in \delta$,

$$
\left\|u_{n h}-u_{n}^{h}\right\|_{L_{\Omega}^{\beta}\left(H^{m}\right)} \leq C h^{\alpha} .
$$

Proof. The proof is a consequence of Lemmas 2.2 and 2.3, and similar to the proof of Theorem 4.1 in [9].

Lemma 2.2. Let $k \in \mathbb{N}$ and $u_{0} \in H^{k}$. There exists a (deterministic) positive constant $C_{k}$, which depends only on $g, k$ and $\left\|u_{0}\right\|_{H^{k}}$, and such that almost surely and for all $(n, h) \in \delta$,

$$
\left\|Z_{n}^{h} Z_{n-1}^{h} \cdots Z_{1}^{h} u_{0}\right\|_{H^{k}}=\left\|u_{n}^{h}\right\|_{H^{k}} \leq C_{k} .
$$

Proof. The proof is similar to the proof of Lemma 4.4 in [9] and uses (28).

Lemma 2.3. If $u_{0} \in H^{m+\sigma}$ then there exists a constant $C$ such that for every $\left(t_{0}, t\right) \in \Delta$,

$$
\left\|S\left(t_{0}, t\right) u_{t_{0}}-Z\left(t_{0}, t\right) u_{t_{0}}\right\|_{L_{\Omega}^{\beta}\left(H^{m}\right)} \leq C\left(t-t_{0}\right)^{1+\alpha} .
$$

Proof. We have

$$
\begin{aligned}
S\left(t_{0}, t\right) u_{t_{0}}-Z\left(t_{0}, t\right) u_{t_{0}}= & \int_{t_{0}}^{t} X(\theta, t)\left\{g\left(S\left(t_{0}, \theta\right) u_{t_{0}}\right)-g\left(Z\left(t_{0}, \theta\right) u_{t_{0}}\right)\right\} d \theta \\
& +\int_{t_{0}}^{t} X(\theta, t) g\left(Z\left(t_{0}, \theta\right) u_{t_{0}}\right)-g\left(Z\left(t_{0}, \theta\right) u_{t_{0}}\right) d \theta \\
& +\int_{t_{0}}^{t}\left(g\left(Z\left(t_{0}, \theta\right) u_{t_{0}}\right)-g\left(Y\left(\theta-t_{0}\right) X\left(t_{0}, t\right) u_{t_{0}}\right)\right) d \theta .
\end{aligned}
$$

By the Cauchy-Schwarz inequality, (8), Lemmas 4.8, 4.6 and 4.9, we get

$$
\begin{aligned}
\| S\left(t_{0}, t\right) u_{t_{0}}- & Z\left(t_{0}, t\right) u_{t_{0}} \|_{L_{\Omega}^{\beta}\left(H^{m}\right)}^{\beta} \\
\leq & C\left(t-t_{0}\right)^{\beta-1} \int_{t_{0}}^{t}\left\|S\left(t_{0}, \theta\right) u_{t_{0}}-Z\left(t_{0}, \theta\right) u_{t_{0}}\right\|_{L_{\Omega}^{\beta}\left(H^{m}\right)}^{\beta} d \theta \\
& +C\left(t-t_{0}\right)^{\beta(1+\alpha)} .
\end{aligned}
$$

The proof is completed with the Gronwall lemma.

\section{Local error}

The local error estimate given in Lemma 2.3 is a crucial step of the proof of Theorem 2.1. In this section we establish an expansion of the local error. As a consequence, in Section 5 we improve the error estimate of Theorem 2.1 if $W$ has independent increments and mean zero. 
Throughout the paper we use the notation

$$
\left\langle(\nabla g) \circ \phi_{0} \mid \phi_{1}\right\rangle=\left(\frac{\partial g}{\partial \Re z} \circ \phi_{0}\right) \Re \phi_{1}+\left(\frac{\partial g}{\partial \Im z} \circ \phi_{0}\right) \Im \phi_{1}
$$

for all $\phi_{0}$ and $\phi_{1} \in L^{2}$, where $\frac{\partial g}{\partial \Re z}$ and $\frac{\partial g}{\partial \Im z}$ are the first partial derivatives of $g$. Notice that $\left\langle(\nabla g) \circ \phi_{0} \mid \phi_{1}\right\rangle$ is then in $L^{2}$.

For every $v \in H^{2}$ we define

$$
\mathcal{G} v=A(g \circ v)-\langle(\nabla g) \circ v \mid A v\rangle .
$$

We fix a real number $\beta \geq 1$. In this section we assume that $W$ is a stochastic process with continuous sample paths and finite moments of order $2 \beta$, and such that there exist $\alpha \in(0,1]$ and $C>0$ such that for all $(s, t)$,

$$
\mathbb{E}\left[|W(t)-W(s)|^{2 \beta}\right] \leq C|t-s|^{2 \beta \alpha} .
$$

As a consequence, by the Cauchy-Schwarz inequality we also have

$$
\mathbb{E}\left[|W(t)-W(s)|^{\beta}\right] \leq \sqrt{C}|t-s|^{\beta \alpha} \text { and } \mathbb{E}\left[(W(t)-W(s))^{2}\right] \leq \sqrt[\beta]{C}|t-s|^{2 \alpha} .
$$

For all $\left(t_{0}, t\right) \in \Delta$ we define

$$
I\left(t_{0}, t\right)=\int_{t_{0}}^{t}(W(t)-W(\theta)) d \theta
$$

The following theorem establishes an expansion of the local error.

Theorem 3.1. Let $m \in \mathbb{N}$. If $u_{0} \in H^{m+2 \sigma}$ then there exists a constant $C>0$ such that for every $\left(t_{0}, t\right) \in \Delta$,

$$
\left\|S\left(t_{0}, t\right) u_{t_{0}}-Z\left(t_{0}, t\right) u_{t_{0}}-I\left(t_{0}, t\right) \mathcal{G} u_{t_{0}}\right\|_{L_{\Omega}^{\beta}\left(H^{m}\right)} \leq C\left(t-t_{0}\right)^{2 \alpha+1} .
$$

Hence, the local error $S_{n}^{h} u_{(n-1) h}-Z_{n}^{h} u_{(n-1) h}$ can be approximated by $I_{n}^{h} \mathcal{G} u_{(n-1) h}$ where $I_{n}^{h}=I((n-1) h, n h)$. Notice that in the case of a white noise dispersion (if $W$ is a Brownian motion), we have

$$
\mathbb{E}\left[I_{n}^{h} \mathcal{G} u_{(n-1) h}\right]=0
$$

because the Brownian motion has independent increments with mean zero. This is important in Section 5 in order to improve the order estimate of the scheme.

\section{Proof of Theorem 3.1}

In Subsection 4.1 we present the main steps of the proof of Theorem 3.1. Technical Lemmas are postponed to Subsection 4.2. 


\subsection{Main steps of the proof of Theorem 3.1}

For all $\phi_{1}$ and $\phi_{2}$ in $L^{2}$, we define

$$
R\left(\phi_{1}, \phi_{2}\right)=g \circ \phi_{2}-g \circ \phi_{1}-\left\langle(\nabla g) \circ \phi_{1} \mid \phi_{2}-\phi_{1}\right\rangle .
$$

Hence, for every $v \in L^{2}$ and every $\left(t_{0}, t\right) \in \Delta$,

$$
\begin{aligned}
S\left(t_{0}, t\right) v-Z\left(t_{0}, t\right) v= & \int_{t_{0}}^{t}(X(\theta, t)-\mathrm{Id})(g \circ v) d \theta \\
& +\int_{t_{0}}^{t}\left\langle(\nabla g) \circ v \mid S\left(t_{0}, \theta\right) v-Y\left(\theta-t_{0}\right) X\left(t_{0}, t\right) v\right\rangle d \theta \\
& +\int_{t_{0}}^{t}(X(\theta, t)-\mathrm{Id})\left\langle(\nabla g) \circ v \mid S\left(t_{0}, \theta\right) v-v\right\rangle d \theta \\
& +\int_{t_{0}}^{t} X(\theta, t) R\left(v, S\left(t_{0}, \theta\right) v\right) d \theta-\int_{t_{0}}^{t} R\left(v, Y\left(\theta-t_{0}\right) X\left(t_{0}, t\right) v\right) d \theta
\end{aligned}
$$

The proof of Theorem 3.1 is based on lemmas dealing with the terms of (17).

We fix $m \in \mathbb{N}$ and recall that we assume that $u_{0} \in H^{m+2 \sigma}$. For simplicity, in this subsection the function $u_{t_{0}}$ is denoted by $v$, and the letter $C$ stands for a (deterministic) positive constant which is independent of $\left(t_{0}, t\right)$ and may change from line to line.

Lemma 4.1. There exists a constant $C$ such that for every $\left(t_{0}, t\right) \in \Delta$,

$$
\left\|\int_{t_{0}}^{t}(X(\theta, t)-\mathrm{Id})(g \circ v) d \theta-I\left(t_{0}, t\right) A(g \circ v)\right\|_{L_{\Omega}^{\beta}\left(H^{m}\right)} \leq C\left(t-t_{0}\right)^{1+2 \alpha} .
$$

Proof. Using the Cauchy-Schwarz inequality and Lemma 4.6, we get

$$
\begin{aligned}
& \left\|\int_{t_{0}}^{t}(X(\theta, t)-\mathrm{Id})(g \circ v) d \theta-I\left(t_{0}, t\right) A(g \circ v)\right\|_{H^{m}} \\
& \quad \leq \int_{t_{0}}^{t}\|(X(\theta, t)-\mathrm{Id})(g \circ v)-(W(t)-W(\theta)) A(g \circ v)\|_{H^{m}} d \theta \\
& \quad \leq\left\|A^{2}(g \circ v)\right\|_{H^{m}} \int_{t_{0}}^{t}|W(t)-W(\theta)|^{2} d \theta \leq C \int_{t_{0}}^{t}|W(t)-W(\theta)|^{2} d \theta .
\end{aligned}
$$

Then Lemma 4.9 and (12) complete the proof.

Lemma 4.2. There exists a constant $C$ such that for every $\left(t_{0}, t\right) \in \Delta$,

$$
\begin{gathered}
\left\|\int_{t_{0}}^{t}\left\langle(\nabla g) \circ v \mid S\left(t_{0}, \theta\right) v-Y\left(\theta-t_{0}\right) X\left(t_{0}, t\right) v\right\rangle d \theta+I\left(t_{0}, t\right)\langle(\nabla g) \circ v \mid A v\rangle\right\|_{L_{\Omega}^{\beta}\left(H^{m}\right)}^{\beta} \\
\leq C\left(\left(t-t_{0}\right)^{\beta(1+2 \alpha)}+\left(t-t_{0}\right)^{\beta-1} \int_{t_{0}}^{t}\left\|S\left(t_{0}, \theta\right) v-Z\left(t_{0}, \theta\right) v\right\|_{L_{\Omega}^{\beta}\left(H^{m}\right)}^{\beta} d \theta\right) .
\end{gathered}
$$


Proof. We have

$$
\begin{aligned}
\int_{t_{0}}^{t}\langle(\nabla g) & \circ v\left|S\left(t_{0}, \theta\right) v-Y\left(\theta-t_{0}\right) X\left(t_{0}, t\right) v\right\rangle d \theta+I\left(t_{0}, t\right)\langle(\nabla g) \circ v \mid A v\rangle \\
= & \int_{t_{0}}^{t}\left\langle(\nabla g) \circ v \mid X\left(t_{0}, \theta\right) v-X\left(t_{0}, t\right) v\right\rangle d \theta+I\left(t_{0}, t\right)\langle(\nabla g) \circ v \mid A v\rangle \\
& +\int_{t_{0}}^{t}\left\langle(\nabla g) \circ v \mid\left(Y\left(\theta-t_{0}\right)-\mathrm{Id}\right) X\left(t_{0}, \theta\right) v-\left(Y\left(\theta-t_{0}\right)-\mathrm{Id}\right) X\left(t_{0}, t\right) v\right\rangle d \theta \\
& +\int_{t_{0}}^{t}\left\langle(\nabla g) \circ v \mid S\left(t_{0}, \theta\right) v-Z\left(t_{0}, \theta\right) v\right\rangle d \theta .
\end{aligned}
$$

We then study the terms of the right-hand side of (18). From the Sobolev inequality $\left\|\phi_{1}\right\|_{L^{\infty}} \leq c\|\phi\|_{H^{1}}$ (for all $\phi \in H^{1}$ ), there exists $c_{m}$ (which depends on $m$ ) such that for all $\phi_{1} \in H^{m+1}$ and $\phi_{2} \in H^{m}$,

$$
\left\|\phi_{1} \phi_{2}\right\|_{H^{m}} \leq c_{m}\left\|\phi_{1}\right\|_{H^{m+1}}\left\|\phi_{2}\right\|_{H^{m}} .
$$

Then by (19) and Lemma 4.6, we have

$$
\begin{aligned}
& \| \int_{t_{0}}^{t}\langle(\nabla g) \circ v\left|X\left(t_{0}, \theta\right) v-X\left(t_{0}, t\right) v\right\rangle d \theta+\langle(\nabla g) \circ v \mid A v\rangle I\left(t_{0}, t\right) \|_{H^{m}} \\
& \quad \leq \int_{t_{0}}^{t}\left\|\left\langle(\nabla g) \circ v \mid X\left(t_{0}, \theta\right) v-X\left(t_{0}, t\right) v+(W(t)-W(\theta)) A v\right\rangle\right\|_{H^{m}} d \theta \\
& \quad \leq C\|(\nabla g) \circ v\|_{H^{m+1}} \int_{t_{0}}^{t}\left\|X\left(t_{0}, \theta\right) v-X\left(t_{0}, t\right) v+(W(t)-W(\theta)) A v\right\|_{H^{m}} d \theta \\
& \quad \leq C\left\|A^{2} v\right\|_{H^{m}} \int_{t_{0}}^{t}|W(t)-W(\theta)| \sqrt{|W(t)-W(\theta)|^{2}+\left|W(\theta)-W\left(t_{0}\right)\right|^{2}} d \theta .
\end{aligned}
$$

Then by Lemma 4.9 and (11) we get

$$
\begin{aligned}
\left\|\int_{t_{0}}^{t}\left\langle(\nabla g) \circ v \mid X\left(t_{0}, \theta\right) v-X\left(t_{0}, t\right) v\right\rangle d \theta+\langle(\nabla g) \circ v \mid A v\rangle I\left(t_{0}, t\right)\right\|_{L_{\Omega}^{\beta}\left(H^{m}\right)} \\
\leq C\left(t-t_{0}\right)^{1+2 \alpha} .
\end{aligned}
$$

By (19) and Lemma 4.9, we also have

$$
\begin{aligned}
& \left\|\int_{t_{0}}^{t}\left\langle(\nabla g) \circ v \mid\left(Y\left(\theta-t_{0}\right)-\mathrm{Id}\right) X\left(t_{0}, \theta\right) v-\left(Y\left(\theta-t_{0}\right)-\mathrm{Id}\right) X\left(t_{0}, t\right) v\right\rangle d \theta\right\|_{L_{\Omega}^{\beta}\left(H^{m}\right)} \\
& \quad \leq C \int_{t_{0}}^{t}\left\|\left(Y\left(\theta-t_{0}\right)-\mathrm{Id}\right) X\left(t_{0}, \theta\right) v-\left(Y\left(\theta-t_{0}\right)-\mathrm{Id}\right) X\left(t_{0}, t\right) v\right\|_{L_{\Omega}^{\beta}\left(H^{m}\right)} d \theta \\
& \quad \leq C \int_{t_{0}}^{t}\left\|\int_{t_{0}}^{\theta}\left(g\left(Y\left(\sigma-t_{0}\right) X\left(t_{0}, \theta\right) v\right)-g\left(Y\left(\sigma-t_{0}\right) X\left(t_{0}, t\right) v\right)\right) d \sigma\right\|_{L_{\Omega}^{\beta}\left(H^{m}\right)} d \theta \\
& \quad \leq C\left(t-t_{0}\right) \int_{t_{0}}^{t}\left\|X\left(t_{0}, \theta\right) v-X\left(t_{0}, t\right) v\right\|_{L_{\Omega}^{\beta}\left(H^{m}\right)} d \theta \\
& \quad \leq C\left(t-t_{0}\right)^{(2+\alpha)} \leq C\left(t-t_{0}\right)^{(1+2 \alpha)},
\end{aligned}
$$


and

$$
\begin{aligned}
& \left\|\int_{t_{0}}^{t}\left\langle(\nabla g) \circ v \mid S\left(t_{0}, \theta\right) v-Z\left(t_{0}, \theta\right) v\right\rangle d \theta\right\|_{L_{\Omega}^{\beta}\left(H^{m}\right)}^{\beta} \\
& \leq C\left(t-t_{0}\right)^{\beta-1} \int_{t_{0}}^{t}\left\|S\left(t_{0}, \theta\right) v-Z\left(t_{0}, \theta\right) v\right\|_{L_{\Omega}^{\beta}\left(H^{m}\right)}^{\beta} d \theta .
\end{aligned}
$$

By (18), (20), (21) and (22), we prove the lemma.

Lemma 4.3. There exists a constant $C$ such that for every $\left(t_{0}, t\right) \in \Delta$,

$$
\left\|\int_{t_{0}}^{t}(X(\theta, t)-\mathrm{Id})\left\langle(\nabla g) \circ v \mid S\left(t_{0}, \theta\right) v-v\right\rangle d \theta\right\|_{L_{\Omega}^{\beta}\left(H^{m}\right)} \leq C\left(t-t_{0}\right)^{1+2 \alpha} .
$$

Proof. By Lemma 4.6, (19) and Lemma 4.9, we have

$$
\begin{aligned}
\| \int_{t_{0}}^{t}(X(\theta, t) & -\mathrm{Id})\left\langle(\nabla g) \circ v \mid S\left(t_{0}, \theta\right) v-v\right\rangle d \theta \|_{H^{m}} \\
& \leq \int_{t_{0}}^{t}|W(t)-W(\theta)|\left\|\left\langle(\nabla g) \circ v \mid S\left(t_{0}, \theta\right) v-v\right\rangle\right\|_{H^{m+\sigma}} d \theta \\
& \leq C\|(\nabla g) \circ v\|_{H^{m+\sigma+1}} \int_{t_{0}}^{t}|W(t)-W(\theta)|\left\|S\left(t_{0}, \theta\right) v-v\right\|_{H^{m+\sigma}} d \theta \\
& \leq C\left(t-t_{0}\right)^{\beta-1} \int_{t_{0}}^{t}|W(t)-W(\theta)|^{\beta}\left\|S\left(t_{0}, \theta\right) v-v\right\|_{H^{m+\sigma}}^{\beta} d \theta .
\end{aligned}
$$

From Lemmas 4.10 and 4.9 and (11), we deduce

$$
\begin{aligned}
& \left\|\int_{t_{0}}^{t}(X(\theta, t)-\mathrm{Id})\left\langle(\nabla g) \circ v \mid S\left(t_{0}, \theta\right) v-v\right\rangle d \theta\right\|_{L_{\Omega}^{\beta}\left(H^{m}\right)}^{\beta} \\
& \quad \leq C\left(t-t_{0}\right)^{\beta-1} \int_{t_{0}}^{t} \mathbb{E}\left[(W(t)-W(\theta))^{\beta}\left(\left(W(\theta)-W\left(t_{0}\right)\right)^{2}+\left(\theta-t_{0}\right)^{2}\right)^{\beta / 2}\right] d \theta \\
& \quad \leq C\left(t-t_{0}\right)^{\beta \min \{1+2 \alpha ; 2+\alpha\}} \leq C\left(t-t_{0}\right)^{\beta(1+2 \alpha)},
\end{aligned}
$$

which completes the proof.

Lemma 4.4. There exists a constant $C$ such that for every $\left(t_{0}, t\right) \in \Delta$,

$$
\left\|\int_{t_{0}}^{t} X(\theta, t) R\left(v, S\left(t_{0}, \theta\right) v\right) d \theta\right\|_{L_{\Omega}^{\beta}\left(H^{m}\right)} \leq C\left(t-t_{0}\right)^{1+2 \alpha} .
$$

Proof. We have

$$
\begin{aligned}
\left\|\int_{t_{0}}^{t} X(\theta, t) R\left(v, S\left(t_{0}, \theta\right) v\right) d \theta\right\|_{H^{m}} & \leq C \int_{t_{0}}^{t}\left\|\left(S\left(t_{0}, \theta\right) v-v\right)^{2}\right\|_{H^{m}} d \theta \\
& \leq C \int_{t_{0}}^{t}\left\|S\left(t_{0}, \theta\right) v-v\right\|_{H^{m+1}}\left\|S\left(t_{0}, \theta\right) v-v\right\|_{H^{m}} d \theta .
\end{aligned}
$$


By Lemma 4.10, we have

$$
\left\|S\left(t_{0}, \theta\right) v-v\right\|_{H^{m+1}}\left\|S\left(t_{0}, \theta\right) v-v\right\|_{H^{m}} \leq C\left(\left|W(\theta)-W\left(t_{0}\right)\right|^{2}+\left|\theta-t_{0}\right|^{2}\right)
$$

and by (11) we prove the lemma.

Lemma 4.5. There exists a constant $C$ such that for every $\left(t_{0}, t\right) \in \Delta$,

$$
\left\|\int_{t_{0}}^{t} R\left(v, Y\left(\theta-t_{0}\right) X\left(t_{0}, t\right) v\right) d \theta\right\|_{L_{\Omega}^{\beta}\left(H^{m}\right)} \leq C\left(t-t_{0}\right)^{1+2 \alpha} .
$$

Proof. We have

$$
\left\|\int_{t_{0}}^{t} R\left(v, Y\left(\theta-t_{0}\right) X\left(t_{0}, t\right) v\right) d \theta\right\|_{H^{m}} \leq C \int_{t_{0}}^{t}\left\|\left(Y\left(\theta-t_{0}\right) X\left(t_{0}, t\right) v-v\right)^{2}\right\|_{H^{m}} d \theta .
$$

The remainder of the proof is similar to the proof of Lemma 4.4.

Finally, we give the proof of Theorem 3.1.

Proof. (Theorem 3.1) By (17) and Lemmas 4.1, 4.2, 4.3, 4.4 and 4.5, there exists a constant $C$ such that for every $\left(t_{0}, t\right) \in \Delta$,

$$
\begin{aligned}
& \left\|S\left(t_{0}, t\right) v-Z\left(t_{0}, t\right) v-I\left(t_{0}, t\right) \mathcal{G} v\right\|_{L_{\Omega}^{\beta}\left(H^{m}\right)}^{\beta} \\
& \quad \leq C\left(\left(t-t_{0}\right)^{\beta(1+2 \alpha)}+\left(t-t_{0}\right)^{\beta-1} \int_{t_{0}}^{t}\left\|S\left(t_{0}, \theta\right) v-Z\left(t_{0}, \theta\right) v\right\|_{L_{\Omega}^{\beta}\left(H^{m}\right)}^{\beta} d \theta\right) .
\end{aligned}
$$

Moreover, because of Lemma 2.3, we have

$$
\left\|S\left(t_{0}, t\right) v-Z\left(t_{0}, t\right) v\right\|_{L_{\Omega}^{\beta}\left(H^{m}\right)}^{\beta} \leq C\left(t-t_{0}\right)^{\beta(1+\alpha)}
$$

and then, with (24),

$$
\mathbb{E}\left[\left\|S\left(t_{0}, t\right) v-Z\left(t_{0}, t\right) v-I\left(t_{0}, t\right) \mathcal{G} v\right\|_{H^{m}}\right] \leq C\left(t-t_{0}\right)^{1+2 \alpha},
$$

which completes the proof.

\subsection{Technical lemmas}

Throughout this subsection, $m$ a positive integer and, unless otherwise mentioned, $W$ is a general real continuous function.

Lemma 4.6. If $v \in H^{m+\sigma}$ then for every $\left(t_{1}, t_{2}\right) \in \Delta$,

$$
\left\|\left(X\left(t_{1}, t_{2}\right)-\mathrm{Id}\right) v\right\|_{H^{m}} \leq\left|W\left(t_{2}\right)-W\left(t_{1}\right)\right|\|v\|_{H^{m+\sigma}} .
$$

If $v \in H^{m+2 \sigma}$ then for all $t_{0}, t_{1}$ and $t_{2}$ such that $t_{0} \leq t_{1} \leq t_{2}$, we have

$$
\begin{aligned}
& \left\|\left(X\left(t_{0}, t_{2}\right)-X\left(t_{0}, t_{1}\right)\right) v-\left(W\left(t_{2}\right)-W\left(t_{1}\right)\right) A v\right\|_{H^{m}}^{2} \\
& \quad \leq\left|W\left(t_{2}\right)-W\left(t_{1}\right)\right|^{2}\left(\left|W\left(t_{2}\right)-W\left(t_{1}\right)\right|^{2}+\left|W\left(t_{1}\right)-W\left(t_{0}\right)\right|^{2}\right)\|v\|_{H^{m+2 \sigma}}^{2} .
\end{aligned}
$$

In particular, if $t_{0}=t_{1}$ then

$$
\left\|\left(X\left(t_{1}, t_{2}\right)-\mathrm{Id}\right) v-\left(W\left(t_{2}\right)-W\left(t_{1}\right)\right) A v\right\|_{H^{m}} \leq\left|W\left(t_{2}\right)-W\left(t_{1}\right)\right|^{2}\|v\|_{H^{m+2 \sigma}} .
$$


Proof. We assume that $v \in H^{m+2 \sigma}$. If we define $r: z \mapsto e^{i z}-1-i z$ then we can write

$$
\begin{aligned}
&\left(X\left(t_{1}, t_{2}\right)-\mathrm{Id}\right) v-\left(W\left(t_{2}\right)-W\left(t_{1}\right)\right) A v \\
& \quad=\mathcal{F}^{-1}\left(\xi \mapsto r\left(a(\xi)\left(W\left(t_{2}\right)-W\left(t_{1}\right)\right)\right) \mathcal{F}(v)(\xi)\right) .
\end{aligned}
$$

Because of (2), we get

$$
\left\|\left(X\left(t_{1}, t_{2}\right)-\mathrm{Id}\right) v-\left(W\left(t_{2}\right)-W\left(t_{1}\right)\right) A v\right\|_{H^{m}}^{2} \leq\left|W\left(t_{2}\right)-W\left(t_{1}\right)\right|^{4}\|v\|_{H^{m+2 \sigma}}^{2},
$$

which gives (27). The proof of (25) is similar to the proof of (27). We have

$$
\begin{aligned}
&\left\|\left(X\left(t_{0}, t_{2}\right)-X\left(t_{0}, t_{1}\right)\right) v-\left(W\left(t_{2}\right)-W\left(t_{1}\right)\right) A v\right\|_{H^{m}}^{2} \\
& \leq\left\|\left(X\left(t_{0}, t_{2}\right)-X\left(t_{0}, t_{1}\right)\right) v-\left(W\left(t_{2}\right)-W\left(t_{1}\right)\right) A X\left(t_{0}, t_{1}\right) v\right\|_{H^{m}}^{2} \\
&\left.+\mid W\left(t_{2}\right)-W\left(t_{1}\right)\right)\left.\right|^{2}\left\|\left(X\left(t_{0}, t_{1}\right)-\mathrm{Id}\right) A v\right\|_{H^{m}}^{2}
\end{aligned}
$$

By (25) and (27) we get (26).

Lemma 4.7. Let $p \in \mathbb{N}^{*}, v \in H^{p}$, and $f: \mathbb{R}^{2} \rightarrow \mathbb{R}$ be a $p$ times differentiable function such that $f(0)=0$ and $\nabla^{k} f$ is bounded for all $k \in\{1, \cdots, p\}$. Then there exists a constant $C_{p-1}>0$, which depends only on $f, p$ and $\|v\|_{H^{p-1}}$, and such that

$$
\|f \circ v\|_{H^{p}} \leq C_{p-1}\|v\|_{H^{p}} .
$$

Proof. Since $f(0)=0$, we have $\|f \circ v\|_{L^{2}} \leq\|f\|_{\infty}\|v\|_{L^{2}}$. Let $p \geq 1$. By differentiating $p$ times, we get

$$
\left\|\partial_{x}^{p}(f \circ v)\right\|_{L^{2}} \leq C\|\nabla f\|_{\infty}\left\|\partial_{x}^{p} v\right\|_{L^{2}}+C \sum_{k=2}^{p}\left\|\nabla^{k} f\right\|_{\infty} \sum_{\left(l_{1}, \cdots, l_{k}\right) \in \pi(k, p)}\left\|\prod_{r=1}^{k} \partial_{x}^{l_{r}} v\right\|_{L^{2}}
$$

where

$$
\pi(k, p)=\left\{\left(l_{1}, \cdots, l_{k}\right) \mid 1 \leq l_{1} \leq \cdots \leq l_{k} \leq p-1, l_{1}+\cdots+l_{k}=p\right\}
$$

and $C$ is a constant which is independent of $f$ and $v$, and may change from line to line. By (19), we get

$$
\begin{aligned}
\left\|\partial_{x}^{p}(f \circ v)\right\|_{L^{2}} & \leq C\|\nabla f\|_{\infty}\left\|\partial_{x}^{p} v\right\|_{L^{2}}+C \sum_{k=2}^{p}\left\|\nabla^{k} f\right\|_{\infty} \sum_{\left(l_{1}, \cdots, l_{k}\right) \in \pi(k, p)}\|v\|_{H^{l_{1}}} \prod_{r=2}^{k}\|v\|_{H^{l_{r}+1}} \\
& \leq C\left(\|\nabla f\|_{\infty}+\sum_{k=2}^{p}\left\|\nabla^{k} f\right\|_{\infty} \sum_{\left(l_{1}, \cdots, l_{k}\right) \in \pi(k, p)}\|v\|_{H^{l_{1}}} \prod_{r=2}^{k-1}\|v\|_{H^{l_{r}+1}}\right)\|v\|_{H^{p}} \\
& \leq C\left(\|\nabla f\|_{\infty}+\sum_{k=2}^{p}\left\|\nabla^{k} f\right\|_{\infty}\|v\|_{H^{p-1}}^{k-1}\right)\|v\|_{H^{p}}
\end{aligned}
$$

where $\prod_{r=2}^{1} \cdots=1$. This completes the proof. 
Lemma 4.8. Let $p \in \mathbb{N}^{*}, v_{1}, v_{2} \in H^{p}$, and $f: \mathbb{R}^{2} \rightarrow \mathbb{R}$ be a $p$ times differentiable function such that $f(0)=0$ and $\nabla^{k} f$ is bounded for all $k \in\{1, \cdots, p+1\}$. Then there exists a constant $C_{p}>0$, which depends only on $f, p,\left\|v_{1}\right\|_{H^{p}}$ and $\left\|v_{2}\right\|_{H^{p}}$, and such that

$$
\left\|f \circ v_{1}-f \circ v_{2}\right\|_{H^{p}} \leq C_{p}\left\|v_{1}-v_{2}\right\|_{H^{p}} .
$$

Proof. We use the same notation as in the proof of Lemma 4.7. Moreover, we denote the vector $\left(\partial_{x}^{l_{1}} v, \cdots, \partial_{x}^{l_{k}} v\right)$ by $\left(\partial_{x}^{l_{*}} v\right)_{k}$ for $v=v_{1}$ or $v=v_{2}$. Differentiating $p$ times, we get

$$
\begin{aligned}
\| \partial_{x}^{p}\left(f \circ v_{1}-\right. & \left.f \circ v_{2}\right)\left\|_{L^{2}} \leq\right\|(\nabla f)\left(v_{1}\right)\left(\partial_{x}^{p} v_{1}\right)-(\nabla f)\left(v_{2}\right)\left(\partial_{x}^{p} v_{2}\right) \|_{L^{2}} \\
& +\sum_{k=2}^{p} \sum_{\left(l_{1}, \cdots, l_{k}\right) \in \pi(k, p)}\left\|\left(\nabla^{k} f\right)\left(v_{1}\right)\left(\partial_{x}^{l} v_{1}\right)_{k}-\left(\nabla^{k} f\right)\left(v_{2}\right)\left(\partial_{x}^{l} v_{2}\right)_{k}\right\|_{L^{2}} .
\end{aligned}
$$

Using (19), we get

$$
\begin{aligned}
\left\|(\nabla f)\left(v_{1}\right)\left(\partial_{x}^{p} v_{1}\right)-(\nabla f)\left(v_{2}\right)\left(\partial_{x}^{p} v_{2}\right)\right\|_{L^{2}} \leq & \left\|(\nabla f)\left(v_{1}\right)\left(\partial_{x}^{p} v_{1}\right)-(\nabla f)\left(v_{2}\right)\left(\partial_{x}^{p} v_{1}\right)\right\|_{L^{2}} \\
& +\left\|(\nabla f)\left(v_{2}\right)\left(\partial_{x}^{p} v_{1}\right)-(\nabla f)\left(v_{2}\right)\left(\partial_{x}^{p} v_{2}\right)\right\|_{L^{2}} \\
\leq & \left\|\nabla^{2} f\right\|_{\infty}\left\|v_{1}-v_{2}\right\|_{H^{1}}\left\|v_{1}\right\|_{H^{p}} \\
& +\|\nabla f\|_{\infty}\left\|v_{1}-v_{2}\right\|_{H^{p}} \\
\leq & \left(\left\|\nabla^{2} f\right\|_{\infty}\left\|v_{1}\right\|_{H^{p}}+\|\nabla f\|_{\infty}\right)\left\|v_{1}-v_{2}\right\|_{H^{p}} .
\end{aligned}
$$

For all $k \geq 2$,

$$
\begin{aligned}
\|\left(\nabla^{k} f\right)\left(v_{1}\right)\left(\partial_{x}^{l} \cdot v_{1}\right)_{k}-\left(\nabla^{k} f\right) & \left(v_{2}\right)\left(\partial_{x}^{l \cdot} v_{2}\right)_{k} \|_{L^{2}} \\
\leq & \left\|\left(\nabla^{k} f\right)\left(v_{1}\right)\left(\partial_{x}^{l \cdot} v_{1}\right)_{k}-\left(\nabla^{k} f\right)\left(v_{2}\right)\left(\partial_{x}^{l} \cdot v_{1}\right)_{k}\right\|_{L^{2}} \\
& +\left\|\left(\nabla^{k} f\right)\left(v_{2}\right)\left(\partial_{x}^{l} v_{1}\right)_{k}-\left(\nabla^{k} f\right)\left(v_{2}\right)\left(\partial_{x}^{l} \cdot v_{2}\right)_{k}\right\|_{L^{2}} .
\end{aligned}
$$

By (19), we have

$$
\begin{aligned}
& \left\|\left(\nabla^{k} f\right)\left(v_{1}\right)\left(\partial_{x}^{l} v_{1}\right)_{k}-\left(\nabla^{k} f\right)\left(v_{2}\right)\left(\partial_{x}^{l} v_{1}\right)_{k}\right\|_{L^{2}} \\
& \quad \leq\left\|\nabla^{k+1} f\right\|_{\infty}\left\|v_{1}-v_{2}\right\|_{H^{1}} \prod_{r=1}^{k}\left\|v_{1}\right\|_{H^{l_{r}+1}} \leq\left\|\nabla^{k+1} f\right\|_{\infty}\left\|v_{1}-v_{2}\right\|_{H^{p}}\left\|v_{1}\right\|_{H^{p}}^{k} .
\end{aligned}
$$

Moreover,

$$
\begin{gathered}
\left\|\left(\nabla^{k} f\right)\left(v_{2}\right)\left(\partial_{x}^{l} v_{1}\right)_{k}-\left(\nabla^{k} f\right)\left(v_{2}\right)\left(\partial_{x}^{l} v_{2}\right)_{k}\right\|_{L^{2}} \\
\leq\left\|\nabla^{k} f\right\|_{\infty} \sum_{j=1}^{k}\left\|\partial_{x}^{l_{j}}\left(v_{1}-v_{2}\right)\right\|_{L^{2}} \prod_{r=1}^{j-1}\left\|\partial_{x}^{l_{r}} v_{1}\right\|_{\infty} \prod_{r=j+1}^{k}\left\|\partial_{x}^{l_{r}} v_{2}\right\|_{\infty} \\
\leq\left\|\nabla^{k} f\right\|_{\infty}\left\|v_{1}-v_{2}\right\|_{H^{p}} \sum_{j=1}^{k}\left\|v_{1}\right\|_{H^{p}}^{j-1}\left\|v_{2}\right\|_{H^{p}}^{k-j} .
\end{gathered}
$$

This completes the proof. 
Lemma 4.9. Let $m \in \mathbb{N}$ and $u_{0} \in H^{m}$. There exists a positive constant $C$, which does not depend on $W$, and such that the solution $u$ satisfies for all $t \in[0,1]$,

$$
\left\|u_{t}\right\|_{H^{m}}+\left\|g\left(u_{t}\right)\right\|_{H^{m}}+\left\|(\nabla g)\left(u_{t}\right)\right\|_{H^{m}} \leq C .
$$

Proof. In this proof, $C$ is a constant which does not depend on $W$ and $t$, and may change from line to line. We define the sequence $\left\{v_{k}\right\}_{k \in \mathbb{N}}$ such that $v_{0}=u_{0}$ and for all $k \in \mathbb{N}$,

$$
v_{k+1}(t, x)=X(0, t) u_{0}(x)+\int_{0}^{t} X(\theta, t) g\left(v_{k}(\theta, x)\right) d \theta, \quad(t, x) \in[0,1] \times \mathbb{R} .
$$

We recall that $v_{k} \rightarrow u$ in $\mathcal{C}\left([0,1], L^{2}\right)$ as $k \rightarrow \infty$ (see [9]). Similarly to the proof of Lemma 3.3 of [9] and by using Lemma 4.7, we prove that there exists a constant $C$ such that for all $k$ and $t,\left\|v_{k}(t, \cdot)\right\|_{H^{m}} \leq C$. As in the proof of Lemma 3.6 of [9], we deduce that for all $t,\|u(t, \cdot)\|_{H^{m}} \leq C$. We then prove that for all $t,\|g(u(t, \cdot))\|_{H^{m}} \leq C$ and $\|(\nabla g)(u(t, \cdot))\|_{H^{m}} \leq C$ by using the assumptions on $g$ and Lemma 4.7.

Lemma 4.10. Let $m \in \mathbb{N}$. If $u_{0} \in H^{m}$, then there exists a constant $C$, which does not depend on $W$, and such that for all $\left(t_{1}, t_{2}\right) \in \Delta$,

$$
\left\|Y\left(t_{2}-t_{1}\right) u_{t_{1}}-u_{t_{1}}\right\|_{H^{m}} \leq C\left(t_{2}-t_{1}\right) .
$$

If $v \in H^{m+\sigma}$, then there exists a constant $C$, which does not depend on $W$, and such that for every $\left(t_{1}, t_{2}\right) \in \Delta$,

$$
\left\|Z\left(t_{1}, t_{2}\right) u_{t_{1}}-u_{t_{1}}\right\|_{H^{m}}^{2} \leq C\left(\left|W\left(t_{2}\right)-W\left(t_{1}\right)\right|^{2}+\left|t_{2}-t_{1}\right|^{2}\right)
$$

and

$$
\left\|S\left(t_{1}, t_{2}\right) u_{t_{1}}-u_{t_{1}}\right\|_{H^{m}}^{2} \leq C\left(\left|W\left(t_{2}\right)-W\left(t_{1}\right)\right|^{2}+\left|t_{2}-t_{1}\right|^{2}\right) .
$$

Proof. The proof of (31) is a consequence of Lemma 4.7. We get (32) and (33) by (31) and Lemma 4.6.

\section{Order estimate}

In this section we assume that $W$ is a random process such that for all $t \geq 0$,

$$
W(t)=\int_{0}^{t} \phi(\theta) d B_{\theta}
$$

where $B$ is a standard Brownian motion and $\phi:(0, \infty) \rightarrow \mathbb{R}$ is a (deterministic) function such that there exist $C_{W}>0$ and $b>-1 / 2$ such that for all $t>0$,

$$
|\phi(t)| \leq C_{W} t^{b} .
$$

This ensures that $W(t)$ is well defined for all $t$. We can also deduce that there exists $C>0$ such that for all $(t, s) \in \mathbb{R}^{2}$,

$$
\mathbb{E}\left[(W(t)-W(s))^{2}\right] \leq C|t-s|^{2 \alpha}
$$


where

$$
\alpha=\frac{1}{2} \min \{2 b+1 ; 1\}
$$

Following Theorem 2.1, if $u_{0} \in H^{p+\sigma}$ then there exists a constant $C>0$ such that for all $(n, h) \in \delta$,

$$
\left\|u_{n h}-u_{n}^{h}\right\|_{L_{\Omega}^{2}\left(H^{p}\right)} \leq C h^{\alpha} .
$$

Notice that $W$ has independent increments with mean zero. This enables us to improve (35) by applying Theorem 3.1. We establish that the order of the scheme is at least $2 \alpha$ instead of $\alpha$.

Theorem 5.1. For every $p \in \mathbb{N}$, if $u_{0} \in H^{p+2 \sigma}$ then there exists a constant $C>0$ such that for every $(n, h) \in \delta$,

$$
\left\|u_{n h}-u_{n}^{h}\right\|_{L_{\Omega}^{2}\left(H^{p}\right)} \leq C h^{2 \alpha} .
$$

In this section, the letter $C$ stands for a constant which does not depend on $(n, h)$, unless otherwise mentioned, and may change from line to line.

Proof. Let $p \in \mathbb{N}$. For all $(n, h) \in \delta$ we write

$$
\left\|u_{n h}-u_{n}^{h}\right\|_{H^{p}}^{2}=E_{1}(n, h)+E_{2}(n, h)+2 \mathcal{R} E_{3}(n, h)
$$

where

$$
\begin{aligned}
& E_{1}(n, h)=\left\|S_{n}^{h} u_{(n-1) h}-Z_{n}^{h} u_{(n-1) h}\right\|_{H^{p}}^{2}, E_{2}(n, h)=\left\|Z_{n}^{h} u_{(n-1) h}-Z_{n}^{h} u_{n-1}^{h}\right\|_{H^{p}}^{2}, \\
& \text { and } E_{3}(n, h)=\left\langle S_{n}^{h} u_{(n-1) h}-Z_{n}^{h} u_{(n-1) h}, Z_{n}^{h} u_{(n-1) h}-Z_{n}^{h} u_{n-1}^{h}\right\rangle_{H^{p}},
\end{aligned}
$$

and we define

$$
\mathcal{E}_{n}^{h}:=\mathbb{E}\left[\left\|u_{n h}-u_{n}^{h}\right\|_{H^{p}}^{2}\right]=\left\|u_{n h}-u_{n}^{h}\right\|_{L_{\Omega}^{2}\left(H^{p}\right)}^{2} .
$$

By Lemma 2.3, there exists $C$ such that for every $(n, h) \in \delta$,

$$
\mathbb{E}\left[E_{1}(n, h)\right] \leq C h^{2+2 \alpha} .
$$

By Lemma 5.3, there exists $C$ such that

$$
E_{2}(n, h) \leq \exp (C h)\left\|u_{(n-1) h}-u_{n-1}^{h}\right\|_{H^{p}}^{2},
$$

and, therefore,

$$
\mathbb{E}\left[E_{2}(n, h)\right] \leq \exp (C h) \mathcal{E}_{n-1}^{h} .
$$

From (37), (38) and Lemma 5.2, we deduce

$$
\mathcal{E}_{n}^{h} \leq C h^{2+2 \alpha}+\exp (C h) \mathcal{E}_{n-1}^{h}+C h^{1+2 \alpha} \sqrt{\mathcal{E}_{n-1}^{h}}
$$


and then, because

$$
h^{1+2 \alpha} \sqrt{\mathcal{E}_{n-1}^{h}} \leq C h^{1+4 \alpha}+C h \mathcal{E}_{n-1}^{h},
$$

we get

$$
\mathcal{E}_{n}^{h} \leq C h^{1+4 \alpha}+\exp (C h) \mathcal{E}_{n-1}^{h}
$$

for all $(n, h) \in \delta$. Then we deduce $(36)$.

The remaining part of this section is devoted to lemmas we use in the proof of Theorem 5.1

Lemma 5.2. Let $p \in \mathbb{N}$. With the same notation as in the proof of Theorem 5.1, if $u_{0} \in H^{p+2 \sigma}$ then there exists $C$ such that for all $(n, h) \in \delta$,

$$
\left|\mathbb{E}\left[\mathcal{R} E_{3}(n, h)\right]\right| \leq C h^{1+2 \alpha} \sqrt{\mathcal{E}_{n-1}^{h}} .
$$

Proof. For all $(n, h) \in \delta$, we define

$$
R_{1}(n, h)=S_{n}^{h} u_{(n-1) h}-Z_{n}^{h} u_{(n-1) h}-I_{n}^{h} \mathcal{G} u_{(n-1) h},
$$

and

$$
R_{2}(n, h)=Z_{n}^{h} u_{(n-1) h}-Z_{n}^{h} u_{n-1}^{h}-\left(\operatorname{Id}+W_{n}^{h} A\right)\left(u_{(n-1) h}-u_{n-1}^{h}\right)
$$

where $W_{n}^{h}=W(n h)-W((n-1) h)$. We write

$$
\begin{aligned}
E_{3}(n, h)= & \left\langle I_{n}^{h} \mathcal{G} u_{(n-1) h},\left(\operatorname{Id}+W_{n}^{h} A\right)\left(u_{(n-1) h}-u_{n-1}^{h}\right)\right\rangle_{H^{p}} \\
& +\left\langle I_{n}^{h} \mathcal{G} u_{(n-1) h}, R_{2}(n, h)\right\rangle_{H^{p}} \\
& +\left\langle R_{1}(n, h), Z_{n}^{h} u_{(n-1) h}-Z_{n}^{h} u_{n-1}^{h}\right\rangle_{H^{p}} .
\end{aligned}
$$

From the Cauchy-Schwarz inequality, Theorem 3.1 and Lemma 5.3, there exists $C$ such that

$$
\left|\mathbb{E}\left[\left\langle R_{1}(n, h), Z_{n}^{h} u_{(n-1) h}-Z_{n}^{h} u_{n-1}^{h}\right\rangle_{H^{p}}\right]\right| \leq C h^{1+2 \alpha} \sqrt{\mathcal{E}_{n-1}^{h}}
$$

By the Cauchy-Schwarz inequality and Lemmas 4.6 and 5.4, there exists $C$ such that

$$
\left|\mathbb{E}\left[\left\langle I_{n}^{h} \mathcal{G} u_{(n-1) h}, R_{2}(n, h)\right\rangle_{H^{p}}\right]\right| \leq C h^{1+3 \alpha} \sqrt{\mathcal{E}_{n-1}^{h}} \leq C h^{1+2 \alpha} \sqrt{\mathcal{E}_{n-1}^{h}}
$$

Since Brownian motion has independent increments with mean zero, we obtain

$$
\begin{aligned}
\mid \mathbb{E}\left[\left\langleI_{n}^{h} \mathcal{G} u_{(n-1) h},(\mathrm{Id}\right.\right. & \left.\left.\left.+W_{n}^{h} A\right)\left(u_{(n-1) h}-u_{n-1}^{h}\right)\right\rangle_{H^{p}}\right] \mid \\
& =\left|\mathbb{E}\left[I_{n}^{h} W_{n}^{h}\right] \mathbb{E}\left[\left\langle u_{(n-1) h}, A\left(u_{(n-1) h}-u_{n-1}^{h}\right)\right\rangle_{H^{p}}\right]\right| \\
& =\left|\mathbb{E}\left[I_{n}^{h} W_{n}^{h}\right] \mathbb{E}\left[\left\langle-A u_{(n-1) h}, u_{(n-1) h}-u_{n-1}^{h}\right\rangle_{H^{p}}\right]\right| \\
& \leq C h^{1+2 \alpha} \sqrt{\mathcal{E}_{n-1}^{h}} .
\end{aligned}
$$

As a consequence, we get (39). 
Lemma 5.3. Let $p \in \mathbb{N}$. With the same notation as in the proof of Theorem 5.1, if $u_{0} \in H^{p}$ then there exists $C$ such that almost surely and for all $(n, h) \in \delta$,

$$
\left\|Z_{n}^{h} u_{(n-1) h}-Z_{n}^{h} u_{(n-1)}^{h}\right\|_{H^{p}} \leq \exp (C h)\left\|u_{(n-1) h}-u_{(n-1)}^{h}\right\|_{H^{p}} .
$$

Proof. It suffices to prove that for all $\left(v_{1}, v_{2}\right)$ in $H^{p} \times H^{p}$ there exists a (deterministic) constant $C$, which depends only on $g, p,\left\|v_{1}\right\|_{H^{p}}$ and $\left\|v_{2}\right\|_{H^{p}}$, and such that almost surely and for every $\left(t_{0}, t\right) \in \Delta$,

$$
\left\|Z\left(t_{0}, t\right) v_{1}-Z\left(t_{0}, t\right) v_{2}\right\|_{H^{p}} \leq \exp \left(C\left(t-t_{0}\right)\right)\left\|v_{1}-v_{2}\right\|_{H^{p}}
$$

We have

$$
\begin{aligned}
Z\left(t_{0}, t\right) v_{2}-Z\left(t_{0}, t\right) v_{1}= & X\left(t_{0}, t\right) v_{2}-X\left(t_{0}, t\right) v_{1} \\
& +\int_{t_{0}}^{t}\left(g\left(Y\left(\theta-t_{0}\right) X\left(t_{0}, t\right) v_{2}\right)-g\left(Y\left(\theta-t_{0}\right) X\left(t_{0}, t\right) v_{1}\right)\right) d \theta
\end{aligned}
$$

By Lemma 4.8,

$$
\begin{array}{r}
\left\|\int_{t_{0}}^{t}\left(g\left(Y\left(\theta-t_{0}\right) X\left(t_{0}, t\right) v_{2}\right)-g\left(Y\left(\theta-t_{0}\right) X\left(t_{0}, t\right) v_{1}\right)\right) d \theta\right\|_{H^{p}} \\
\leq C \int_{t_{0}}^{t}\left\|Y\left(\theta-t_{0}\right) X\left(t_{0}, t\right) v_{2}-Y\left(\theta-t_{0}\right) X\left(t_{0}, t\right) v_{1}\right\|_{H^{p}} d \theta
\end{array}
$$

and by the Gronwall lemma we get

$$
\begin{aligned}
\left\|Z\left(t_{0}, t\right) v_{1}-Z\left(t_{0}, t\right) v_{2}\right\|_{H^{p}} & \leq \exp \left(C\left(t-t_{0}\right)\right)\left\|X\left(t_{0}, t\right)\left(v_{1}-v_{2}\right)\right\|_{H^{p}} \\
& =\exp \left(C\left(t-t_{0}\right)\right)\left\|v_{1}-v_{2}\right\|_{H^{p}} .
\end{aligned}
$$

This completes the proof.

Lemma 5.4. Let $p \in \mathbb{N}$. With the same notation as in the proof of Lemma 5.2, if $u_{0} \in H^{p+2 \sigma}$, then there exists a constant $C$ such that for every $(n, h) \in \delta$,

$$
\left\|R_{2}(n, h)\right\|_{L_{\Omega}^{2}\left(H^{p}\right)} \leq C h^{2 \alpha}\left\|u_{n-1}^{h}-u_{(n-1) h}\right\|_{L_{\Omega}^{2}\left(H^{p}\right)} .
$$

Proof. We write

$$
\begin{aligned}
& Z_{n}^{h} u_{n-1}^{h}-Z_{n}^{h} u_{(n-1) h} \\
& \quad=\left(\operatorname{Id}+W_{n}^{h} A\right)\left(u_{n-1}^{h}-u_{(n-1) h}\right)+\left(X_{n}^{h}-\mathrm{Id}-W_{n}^{h} A\right)\left(u_{n-1}^{h}-u_{(n-1) h}\right) \\
& \quad+\int_{(n-1) h}^{n h}\left(g\left(Y(\theta-(n-1) h) X_{n}^{h} u_{n-1}^{h}\right)-g\left(Y(\theta-(n-1) h) X_{n}^{h} u_{(n-1) h}\right)\right) d \theta .
\end{aligned}
$$

The proof is completed by Lemmas 4.6 and 4.8 . 


\section{Another splitting scheme}

\subsection{Setting and results}

In this section we deal with another splitting scheme for Equation (3). Using the same notation as in Section 2.2 we define for all $(n, h) \in \delta$ the (Lie) splitting scheme

$$
\bar{u}_{n}^{h}:=\bar{Z}_{n}^{h} \cdots \bar{Z}_{1}^{h} u_{0}
$$

where

$$
\bar{Z}_{k}^{h}:=\bar{Z}((k-1) h, k h) \text { for all } k \in\{1, \cdots, n\}
$$

and

$$
\bar{Z}\left(t_{0}, t\right):=X\left(t_{0}, t\right) Y\left(t-t_{0}\right) \text { for all }\left(t_{0}, t\right) \in \Delta .
$$

This is another Lie splitting scheme. Under the same assumptions as in Section 2.2 we establish an order estimate for this scheme. The proof is omitted because it is similar to the proof of Theorem 2.1.

Theorem 6.1. Let $m \in \mathbb{N}$. If $u_{0} \in H^{m+\sigma}$ then there exists a constant $C>0$ such that for all $(n, h) \in \delta$,

$$
\left\|u_{n h}-\bar{u}_{n}^{h}\right\|_{L_{\Omega}^{\beta}\left(H^{m}\right)} \leq C h^{\alpha} .
$$

Under the same assumptions as in Section 3 we have the following result that we prove in the next subsection.

Theorem 6.2. Let $m \in \mathbb{N}$ and $W$ be a stochastic process satisfying (11). If $u_{0} \in H^{m+2 \sigma}$ then there exists a constant $C$ such that for every $\left(t_{0}, t\right) \in \Delta$,

$$
\left\|S\left(t_{0}, t\right) u_{t_{0}}-\bar{Z}\left(t_{0}, t\right) u_{t_{0}}+J\left(t_{0}, t\right) \mathcal{G} u_{t_{0}}\right\|_{L_{\Omega}^{\beta}\left(H^{m}\right)} \leq C\left(t-t_{0}\right)^{2 \alpha+1}
$$

where $J\left(t_{0}, t\right)=\int_{t_{0}}^{t}\left(W(\theta)-W\left(t_{0}\right)\right) d \theta$.

Therefore, the local error $S_{n}^{h} u_{(n-1) h}-\bar{Z}_{n}^{h} u_{(n-1) h}$ can be approximated by $-J_{n}^{h} \mathcal{G} u_{(n-1) h}$ where $J_{n}^{h}=J((n-1) h, n h)$.

As a consequence, we have the following result regarding the global error if $W$ is Gaussian and have independent increments and mean zero.

Theorem 6.3. Let $W$ be a process as defined in Section 5. For every $p \in \mathbb{N}$, if $u_{0} \in$ $H^{p+2 \sigma}$ then there exists a constant $C>0$ such that for every $(n, h) \in \delta$,

$$
\left\|u_{n h}-\bar{u}_{n}^{h}\right\|_{L_{\Omega}^{2}\left(H^{p}\right)} \leq C h^{2 \alpha} .
$$

We omit the proof of Theorem 6.3 because it is similar to the proof of Theorem 5.1 with $-J_{n}^{h}$ instead of $I_{n}^{h}$. Notice also that the results of Lemmas 2.3, 5.2, 5.3 and 5.4 still hold if we replace $Z$ with $\bar{Z}$. 


\subsection{Proof of Theorem 6.2}

We write

$$
\begin{aligned}
S\left(t_{0}, t\right) v-\bar{Z}\left(t_{0}, t\right) v= & \int_{t_{0}}^{t}\left(X(\theta, t)-X\left(t_{0}, t\right)\right)(g \circ v) d \theta \\
& +\int_{t_{0}}^{t} X\left(t_{0}, t\right)\left\langle(\nabla g) \circ v \mid S\left(t_{0}, \theta\right) v-Y\left(\theta-t_{0}\right) v\right\rangle d \theta \\
& +\int_{t_{0}}^{t}\left(X(\theta, t)-X\left(t_{0}, t\right)\right)\left\langle(\nabla g) \circ v \mid S\left(t_{0}, \theta\right) v-v\right\rangle d \theta \\
& +\int_{t_{0}}^{t} X(\theta, t) R\left(v, S\left(t_{0}, \theta\right) v\right) d \theta-\int_{t_{0}}^{t} X\left(t_{0}, t\right) R\left(v, Y\left(\theta-t_{0}\right) v\right) d \theta
\end{aligned}
$$

where $v=u_{t_{0}}$. By using the same tools as in the proof of Lemma 4.1 we get

$$
\left\|\int_{t_{0}}^{t}\left(X(\theta, t)-X\left(t_{0}, t\right)\right)(g \circ v) d \theta+J\left(t_{0}, t\right) A(g \circ v)\right\|_{L_{\Omega}^{\beta}\left(H^{m}\right)} \leq C\left(t-t_{0}\right)^{1+2 \alpha} .
$$

In the same way as for Lemma 4.2 we obtain

$$
\begin{gathered}
\left\|\int_{t_{0}}^{t} X\left(t_{0}, t\right)\left\langle(\nabla g) \circ v \mid S\left(t_{0}, \theta\right) v-Y\left(\theta-t_{0}\right) v\right\rangle d \theta-J\left(t_{0}, t\right)\langle(\nabla g) \circ v \mid A v\rangle\right\|_{L_{\Omega}^{\beta}\left(H^{m}\right)}^{\beta} \\
\leq C\left(\left(t-t_{0}\right)^{\beta(1+2 \alpha)}+\left(t-t_{0}\right)^{\beta-1} \int_{t_{0}}^{t}\left\|S\left(t_{0}, \theta\right) v-\bar{Z}\left(t_{0}, \theta\right) v\right\|_{L_{\Omega}^{\beta}\left(H^{m}\right)}^{\beta} d \theta\right) .
\end{gathered}
$$

Similarly to Lemma 4.3 we can prove

$$
\left\|\int_{t_{0}}^{t}\left(X(\theta, t)-X\left(t_{0}, t\right)\right)\left\langle(\nabla g) \circ v \mid S\left(t_{0}, \theta\right) v-v\right\rangle d \theta\right\|_{L_{\Omega}^{\beta}\left(H^{m}\right)} \leq C\left(t-t_{0}\right)^{1+2 \alpha} .
$$

By Lemma 4.4 we have

$$
\left\|\int_{t_{0}}^{t} X(\theta, t) R\left(v, S\left(t_{0}, \theta\right) v\right) d \theta\right\|_{L_{\Omega}^{\beta}\left(H^{m}\right)} \leq C\left(t-t_{0}\right)^{1+2 \alpha}
$$

and as for Lemma 4.5 we get

$$
\left\|\int_{t_{0}}^{t} X\left(t_{0}, t\right) R\left(v, Y\left(\theta-t_{0}\right) v\right) d \theta\right\|_{L_{\Omega}^{\beta}\left(H^{m}\right)} \leq C\left(t-t_{0}\right)^{1+2 \alpha} .
$$

Finally we deduce from above that

$$
\begin{aligned}
\| S\left(t_{0}, t\right) v- & \bar{Z}\left(t_{0}, t\right) v+J\left(t_{0}, t\right) \mathcal{G} v \|_{L_{\Omega}^{\beta}\left(H^{m}\right)}^{\beta} \\
& \leq C\left(\left(t-t_{0}\right)^{\beta(1+2 \alpha)}+\left(t-t_{0}\right)^{\beta-1} \int_{t_{0}}^{t}\left\|S\left(t_{0}, \theta\right) v-\bar{Z}\left(t_{0}, \theta\right) v\right\|_{L_{\Omega}^{\beta}\left(H^{m}\right)}^{\beta} d \theta\right)
\end{aligned}
$$

and we complete the proof as for Theorem 3.1 and by using

$$
\left\|S\left(t_{0}, t\right) v-\bar{Z}\left(t_{0}, t\right) v\right\|_{L_{\Omega}^{\beta}\left(H^{m}\right)} \leq C\left(t-t_{0}\right)^{1+\alpha}
$$

which is proven as Lemma 2.3. 


\section{References}

[1] G. Bal and L. Ryzhik, Time splitting for wave equations in random media, ESAIM: M2AN, 38, pp. 961-987 (2004)

[2] G. Bal and L. Ryzhik, Time splitting for the Liouville equation in a random medium, Commun. Math. Sci., 2, pp. 515-534 (2004)

[3] R. Belaouar, A. De Bouard, A. Debussche, Numerical analysis of the nonlinear Schrödinger equation with white noise dispersion, Stochastic and Partial Differential Equations: Analysis and Computations, 3, pp.103-132 (2015)

[4] C. Besse, B. Bidégaray and S. Descombes, Order estimates in time of splitting methods for the nonlinear Schrödinger equation, SIAM J. Numer. Anal., 40, pp. 26-40 (2003)

[5] D. Cohen and G. Dujardin, Exponential integrators for nonlinear Schrödinger equations with white noise dispersion, Stochastics and Partial Differential Equations: Analysis and Computations, 5, pp. 592-613 (2017)

[6] A. De Bouard and A. Debussche, The nonlinear Schrödinger equation with white noise dispersion, Journal of Functional Analysis, 259, pp. 1300-1321 (2010)

[7] A. Debussche and Y. Tsutsumi, 1D quintic nonlinear Schrödinger equation with white noise dispersion, Journal de Mathématiques Pures et Appliquées, 96, pp. 363-376 (2011)

[8] R. Duboscq and R. Marty, Analysis of a splitting scheme for a class of random nonlinear partial differential equations, ESAIM: PS, 20, pp. 572-589 (2016)

[9] R. Marty, On a splitting scheme for the nonlinear Schrödinger equation in a random medium, Commun. Math. Sci., 4, pp. 679-705 (2006)

[10] G. Strang, On the construction and comparison of difference schemes, SIAM J. Numer. Anal., 5, pp. 506-517 (1968) 